\title{
VALOR DA VARREDURA COM IODO ANTES DA TERAPIA ABLATIVA EM PACIENTES COM CARCINOMA DE TIREÓIDE*
}

\author{
Pedro Weslley Souza do Rosário ${ }^{1}$, Ludmilla David Cardoso², Tales Alvarenga Fagundes², \\ Álvaro Luís Barroso ${ }^{3}$, Leonardo Lamego Rezende ${ }^{4}$, Eduardo Lanza Padrão ${ }^{4}$, Saulo Purisch ${ }^{5}$
}

\begin{abstract}
Resumo OBJETIVO: Avaliar a relevância clínica da varredura pré-dose ablativa em pacientes com carcinoma diferenciado de tireóide. MATERIAIS E MÉTODOS: Analisamos a varredura com ${ }^{131} \mathrm{I}$ e a tireoglobulina $(\mathrm{Tg})$ sérica em hipotireoidismo antes da primeira terapia ablativa em 100 pacientes submetidos a tireoidectomia total, considerando a varredura clinicamente importante quando revelou metástases ressecáveis ou que foram tratadas com doses maiores que a inicialmente proposta $\left(100 \mathrm{mCi}\right.$ de $\left.{ }^{131} \mathrm{I}\right)$, além dos casos sem captação e com $\mathrm{Tg}<5 \mathrm{ng} / \mathrm{ml}$, que não receberam radioiodoterapia. RESULTADOS: A varredura revelou captação correspondente a metástases linfonodais em dez pacientes $(10 \%)$, metástases distantes em cinco $(5 \%)$, apenas em leito tireoidiano em $76(76 \%)$ e foi negativa em nove $(9 \%)$, sendo clinicamente relevante (indicando cirurgia, aumento da dose ou dispensando a radioiodoterapia) em $18 \%$ dos pacientes. Nos pacientes com $\mathrm{Tg}>10 \mathrm{ng} / \mathrm{ml}$ a varredura influenciou a conduta em $41 \%$ dos casos pela presença de metástases, e naqueles com $\mathrm{Tg}<10 \mathrm{ng} / \mathrm{ml}$ em apenas 10\%, na maioria por não receberem radioiodo. CONCLUSÃO: A varredura pré-dose ablativa fornece informacões clinicamente importantes (presença de metástases) em muitos pacientes com $\mathrm{Tg}>10 \mathrm{ng} / \mathrm{ml}$, sendo indicada nesta condição.
\end{abstract}

Unitermos: Câncer de tireóide; Varredura; Terapia ablativa.

Abstract Usefulness of iodine scanning before ablative therapy in patients with thyroid carcinoma.

OBJECTIVE: To evaluate the clinical benefit of pre-ablation scanning in patients with differentiated thyroid carcinoma. MATERIALS AND METHODS: We assessed scannings and serum thyroglobulin $(\mathrm{Tg})$ measurements before the first ablative therapy in $\mathbf{1 0 0}$ patients with hypothyroidism submitted to total thyroidectomy. Scanning was considered clinically significant when it showed resectable metastases or which were treated with higher doses than the initially proposed one $\left(100 \mathrm{mCi}\right.$ of $\left.{ }^{131} \mathrm{I}\right)$, in addition to cases with no uptake and $\mathrm{Tg}$ levels $<5 \mathrm{ng} / \mathrm{ml}$ not requiring radioiodine therapy. RESULTS: Scanning showed uptake corresponding to lymph node metastases in 10 patients $(10 \%)$, distant metastases in $5(5 \%)$, uptake only in the thyroid bed in $76(76 \%)$, and was negative in $9(9 \%)$. The procedure was considered clinically relevant in $18 \%$ of the patients (indicating the need of surgery, dose increase or discontinuation of radioiodine therapy). In patients with $\mathrm{Tg}>10 \mathrm{ng} / \mathrm{ml}$, scanning influenced therapeutic management in $41 \%$ of the cases due to detection of metastases, while in patients with $\mathrm{Tg}<10 \mathrm{ng} / \mathrm{ml}$ scanning influenced therapeutic management in only $10 \%$ of the patients, most of which did not receive radioiodine therapy. CONCLUSION: Scanning provides clinically significant data (presence of metastases) in many patients with $\mathrm{Tg}$ levels $>10 \mathrm{ng} /$ $\mathrm{ml}$ and, therefore, should be used in these cases.

Key words: Thyroid cancer; Scanning; Ablative therapy.

* Trabalho realizado na Clínica de Endocrinologia e no Serviço de Medicina Nuclear da Santa Casa de Belo Horizonte, Belo Horizonte, MG.

1. Médico Assistente da Clínica de Endocrinologia da Santa Casa de Belo Horizonte.

2. Médicos Residentes de Endocrinologia da Santa Casa de Belo Horizonte.

3. Chefe do Serviço de Medicina Nuclear da Santa Casa de Belo Horizonte.

4. Médicos Assistentes do Serviço de Medicina Nuclear da Santa Casa de Belo Horizonte.

5. Chefe da Clínica de Endocrinologia da Santa Casa de Belo Horizonte.

Endereço para correspondência: Dr. Pedro Weslley Souza Rosário. Centro de Estudos e Pesquisa da Clínica de Endocrinologia e Metabologia (CEPCEM). Avenida Francisco Sales, 1111, 5음 andar, ala D, Santa Efigênia. Belo Horizonte, MG, 30150-221. E-mail: pedrorosario@globo.com

Recebido para publicação em 30/7/2004. Aceito, após revisão, em 9/12/2004.

\section{INTRODUÇÃO}

Após tireoidectomia total e antes da radioiodoterapia, pacientes com carcinoma diferenciado de tireóide (CDT) devem ser avaliados quanto à presença de metástases, que, se forem linfonodais ou macrometástases devem ser abordadas cirurgicamen$t^{(1)}$ e se forem não ressecáveis exigem doses maiores de ${ }^{131}$ I do que as prescritas para ablação de remanescentes apenas ${ }^{(2)}$. Além disso, alguns pacientes podem ser dispensados da terapia ablativa por não apresentarem remanescentes tireoidianos detectáveis na varredura e nem tireoglobulina $(\mathrm{Tg})$ elevada, mesmo em hipotireoidismo ${ }^{(1,3-5)}$.
Para identificar esses casos, a varredura pré-terapia combinada à Tg estimulada são os métodos recomendados ${ }^{(1-4)}$. A varredura com ${ }^{131} \mathrm{I}$ apresenta ainda a vantagem de permitir o cálculo da dose a ser administrada ${ }^{(6)}$ e a medida da captação pelo tecido tireoidiano remanescente ou pelas metástases, o que pode influenciar na dose terapêutica ${ }^{(1,2)}$. No entanto, a necessidade da varredura antes da terapia ablativa não é consensual na literatura ${ }^{(1,7)}$, sendo a sensibilidade deste exame, a eficácia de protocolos com altas doses fixas sem varredura pré-dose ${ }^{(7)}$, a possibilidade de comprometimento da captação da dose terapêutica (efeito "stunning") ${ }^{(1,2)}$, o fato de não 
resultar em mudança na conduta na maioria dos pacientes e a tentativa de reduzir $\operatorname{custos}^{(8)}$, argumentos contrários à sua realização rotineira.

O objetivo deste estudo foi avaliar o impacto da varredura pré-terapia ablativa na decisão clínica em pacientes com carcinoma papilífero de tireóide submetidos à tireoidectomia total.

\section{MATERIAIS E MÉTODOS}

Analisamos, retrospectivamente, os resultados da varredura de corpo inteiro com ${ }^{131}$ I e Tg sérica em hipotireoidismo após a tireoidectomia total (com ressecção de linfonodos aparentemente metastáticos) e antes da terapia ablativa em 100 pacientes (74 mulheres e 26 homens; idade variando de 13 a 76 anos, média de 41,6 anos) com carcinoma papilífero de tireóide (tumor > $1,5 \mathrm{~cm}$ em todos $^{(3)}, 45$ com metástases linfonodais e 23 com extensão além da cápsula tireoidiana no anatomopatológico) atendidos em nossa instituição até o ano de 2002, quando a varredura pré-dose era rotineira em nosso serviço. Selecionamos apenas pacientes sem anticorpos anti-Tg.

Consideramos que a varredura prédose foi clinicamente importante quando revelou metástases que podiam ser abordadas cirurgicamente antes da ablação ou que foram tratadas com doses maiores que 3,7 GBq de ${ }^{131} \mathrm{I}$ (dose fixa mais comumente recomendada em nosso meio $\left.{ }^{(9)}\right)$. Também foram relevantes os casos em que a varredura foi limpa e com $\mathrm{Tg}$ estimulada $<5 \mathrm{ng} / \mathrm{ml}$, que não receberam radioiodoterapia $^{(\mathbf{1 , 3}-\mathbf{5})}$.

Este estudo foi aprovado pelo Comitê de Ética em Pesquisas da instituição.

\section{Métodos de imagem}

Varredura diagnóstica foi realizada com dose traçadora de $5 \mathrm{mCi}$ de ${ }^{131} \mathrm{I}$ durante hipotireoidismo após suspensão de L-T4 por cinco semanas e administração de dieta pobre em iodo durante duas semanas precedendo o exame.

Imagens anteriores e posteriores de corpo inteiro foram obtidas 72 horas após administração do iodo. Todos o pacientes foram mapeados ultilizando câmera gama de dupla cabeça. A sensibilidade de ambas as cabeças foi semelhante e em con- formidade com as especificações da National Electrical Manufacturers Association (NEMA). Varreduras foram analisadas por três profissionais especializados em medicina nuclear. Outros métodos de imagem foram usados para confirmar as metástases aparentes na varredura: radiografia, ultra-som e tomografia computadorizada, além de confirmação histológica em casos selecionados e em todos os que foram operados.

\section{Dosagens laboratoriais}

$\mathrm{Tg}$ foi mensurada por ensaio radioimunométrico, com sensibilidade funcional de $0,8 \mathrm{ng} / \mathrm{ml}$, precisão do intra-ensaio de 2,4$6,6 \%$ e interensaio de $5,1-8 \%$, com valores de referência pelo fabricante de 3-42 $\mathrm{ng} / \mathrm{ml}$. Todos os pacientes mostraram níveis de TSH > 30 mIU/l após suspensão de L-T4 depois de quatro semanas.

$\operatorname{TgAc}$ foi determinado por ensaio quimioluminescente, com limites de $1 \mathrm{IU} / \mathrm{ml}$ e precisão intra-ensaio e interensaio de 8,7 e $5,9 \%$, respectivamente, com valores de 2-40 IU/ml.

\section{RESULTADOS}

A varredura pré-terapia revelou captação cervical ectópica e/ou mediastinal sem lesões a distância em dez pacientes (10\%), metástases distantes com ou sem acometimento de linfonodos em cinco (5\%), captação apenas em leito tireoidiano em 76 $(76 \%)$, e foi negativa em nove $(9 \%)$.
Dos pacientes com metástases cervicais e/ou mediastinais, sete puderam ser abordados cirurgicamente; dos casos com varredura limpa, seis apresentavam Tg em hipotireoidismo $<5 \mathrm{ng} / \mathrm{ml}$, não necessitando da terapia ablativa; e todos com metástases distantes receberam a dose de $200 \mathrm{mCi}$ e dois deles tiveram linfonodos metastáticos ressecados. Portanto, a varredura prédose influenciou a conduta terapêutica (cirurgia, aumento da dose ablativa ou não indicação da radioiodoterapia) em $18 \%$ dos pacientes. A captação em leito tireoidiano foi $<5 \%$ em $92 \%$ dos 76 casos.

Os resultados e a conduta nos pacientes com metástases estão apresentados na Tabela 1.

Dos pacientes com metástases aparentes na varredura, 73,3\% tinham Tg estimulada $>10 \mathrm{ng} / \mathrm{ml}$, e naqueles com varredura sem metástases a $\mathrm{Tg}$ foi $<10 \mathrm{ng} / \mathrm{ml} \mathrm{em}$ $83 \%$. Separando os pacientes baseados na $\mathrm{Tg}$, verificamos que naqueles com $\mathrm{Tg}>10$ $\mathrm{ng} / \mathrm{ml}$, que representaram apenas $22 \%$, o resultado da varredura antes da terapia ablativa influenciou a conduta em $41 \%$ dos casos pela presença de metástases, e naqueles com $\mathrm{Tg}<10 \mathrm{ng} / \mathrm{ml}$ em apenas $10 \%$, na maioria por não receberem radioiodo.

\section{DISCUSSÃO}

Este estudo confirma que, mesmo após a tireoidectomia total, a permanência de remanescentes tireoidianos é muito comum, com apenas $6 \%$ dos pacientes não

Tabela 1 Resultados e conduta nos pacientes com metástases detectadas na varredura pré-terapia.

\begin{tabular}{|l|c|l|}
\hline Captação na varredura & Tg $(\mathrm{ng} / \mathrm{ml})$ & \multicolumn{1}{|c|}{ Conduta } \\
\hline Cervical ectópica & 15,2 & Cirurgia \\
Cervical ectópica & 13,8 & Cirurgia \\
Cervical ectópica & 8,0 & Cirurgia \\
Cervical ectópica & 5,2 & Cirurgia \\
Cervical ectópica & 6,8 & Radioiodo (5,5 GBq) \\
Cervical ectópica & 8,5 & Cirurgia \\
Cervical ectópica e mediastinal média & 20,5 & Radioiodo (5,5 GBq) \\
Cervical ectópica e mediastinal alta & 46,0 & Cirurgia \\
Mediastinal alta & 18,2 & Cirurgia \\
Mediastinal & 24,5 & Radioiodo (5,5 GBq) \\
Pulmonar e cervical ectópica & 73,6 & Cirurgia e radioiodo (7,4 GBq) \\
Pulmonar, cervical ectópica e mediastinal & 109,5 & Cirurgia e radioiodo (7,4 GBq) \\
Pulmonar & 43,3 & Radioiodo (7,4 GBq) \\
Pulmonar & 67,0 & Radioiodo (7,4 GBq) \\
Pulmonar, óssea & 286,8 & Radioiodo (7,4 GBq) \\
\hline
\end{tabular}


apresentando restos aparentes na varredura e corroborado pela $\mathrm{Tg}$ estimulada baixa. Estima-se que de $5 \%$ a $10 \%$ dos pacientes com CDT submetidos à tireoidectomia total se enquandrem nesta situação ${ }^{(5)}$ e poderiam ser dispensados da radioiodoterapia $^{(\mathbf{1 , 3 - 5})}$. Verificamos, ainda, que metástases linfonodais em pacientes com carcinoma papilífero podem não ser completamente retiradas na cirurgia inicial, sendo a investigação pós-operatória necessária ${ }^{(10)}$, considerando que a reabordagem cirúrgica é o recomendado para estes $\operatorname{casos}^{(\mathbf{1})}$.

Apesar da varredura pré-dose ser recomendada por muitos autores ${ }^{(\mathbf{1 - 4})}$, algumas críticas são feitas quanto à sua realização rotineira $^{(\mathbf{1 , 7 , 8})}$. A possibilidade de comprometer a captação da dose ablativa (efeito "stunning") é considerada uma limitação ${ }^{(\mathbf{1})}$. Mas, considerando que a real ocorrência e impacto clínico deste fenômeno não está definido ${ }^{(11)}$ e que pode ser evitado com uma dose traçadora de $2 \mathrm{mCi}^{(\mathbf{1 2})}$ ou administrando-se a terapia em até 72 horas $^{(\mathbf{1 3}, \mathbf{1 4})}$, não acreditamos que esta seja uma razão convincente para a não realização da varredura pré-dose.

Por metástases serem incomuns após a tireoidectomia total e ressecção de linfonodos acometidos, o alvo principal da terapia ablativa são os pacientes com remanescentes tireoidianos, para os quais doses de ${ }^{131} \mathrm{I} \geq 75 \mathrm{mCi}$ são eficazes ${ }^{(2)}$. Assim, é previsível que protocolos com altas doses fixas de radioiodo sem varredura pré-terapia resultem em elevada eficácia ${ }^{(7)}$. No entanto, se a varredura é realizada, um pequeno grupo de pacientes pode ser dispensando do radioiodo por apresentar varredura limpa e Tg estimulada baixa ${ }^{(\mathbf{1}, \mathbf{3}-5)}$, $6 \%$ nesta série. Em nosso serviço adotamos o uso de altas doses de ${ }^{131} \mathrm{I}$ para ablação de remanescentes, uma tendência na maioria dos centros em nosso país ${ }^{(\mathbf{9})}$, mas se aceitarmos que a dose de $30 \mathrm{mCi}$ pode ser administrada em pacientes com baixa captação em leito tireoidiano, uma vez que a eficácia nestes casos é bem estabelecida em diversas séries ${ }^{(\mathbf{6}, 15-23)}$, a maioria dos pacientes sem metástases poderia ser tratada com baixas doses, em regime ambulatorial. Em nosso estudo, $92 \%$ dos pacientes com re- manescentes tireoidianos apenas tinham captação < 5\%. Assim, em serviços que consideram o uso da dose de $30 \mathrm{mCi}$ para pacientes com baixa captação cervical, a varredura pré-dose poderia dispensar a maioria dos pacientes da internação e do uso de doses maiores de ${ }^{131} \mathrm{I}$, sendo, portanto, indicada de rotina.

A contribuição mais relevante da varredura em centros que empregam altas doses de ${ }^{131} \mathrm{I}$, independente da captação em leito tireoidiano, é detectar pacientes com metástases, que poderiam ser ressecadas antes da ablação ou serem tratados já na primeira terapia com doses maiores de ${ }^{131} \mathrm{I}^{\mathbf{1}, \mathbf{2})}$. Pela baixa frequência destas após a tireoidectomia total, concluímos que esta contribuição é incomum em pacientes não selecionados, $12 \%$ nesta publicação. Mas quando analisamos um grupo com maior probabilidade de doença metastática ( $\mathrm{Tg}$ > $10 \mathrm{ng} / \mathrm{ml})^{(\mathbf{1 , 3 , 4})}$, ela passa a ser relevante ( $41 \%$ neste estudo).

Concluímos que a varredura pré-terapia ainda tenha um papel importante, sendo recomendada em pacientes com maior probabilidade de apresentar metastáses ( $\mathrm{Tg}$ $>10 \mathrm{ng} / \mathrm{ml}$ ), em centros que empregam altas doses para ablação de remanescentes, independente da captação cervical. Nos serviços que admitem o uso da dose de 30 mCi para pacientes com baixa captação, a varredura pode dispensar a imensa maioria dos casos da internação e deve ser realizada rotineiramente.

\section{REFERÊNCIAS}

1. Mazzaferri EL. NCCN thyroid carcinoma practice guidelines. Oncology 1999;13(Suppl 11A):391442.

2. Society of Nuclear Medicine procedure guideline for therapy of thyroid disease with iodine-131 (sodium iodide). Reston: Society of Nuclear Medicine, 2002.

3. Schlumberger MJ. Papillary and follicular thyroid carcinoma. N Engl J Med 1998;338:297-306.

4. DeGroot LJ, Pacini F. Thyroid neoplasia. In: DeGroot LJ, Hennemann G, eds. Thyroid disease manager. 2003. www.thyroidmanager.org

5. Ringel MD, Ladenson PW. Controversies in the follow-up and management of well-differentiated thyroid cancer. Endocr Relat Cancer 2004;11:97116.

6. Maxon HR III, Englaro EE, Thomas SR, et al. Radioiodine-131 therapy for well-differentiated thyroid cancer - a quantitative radiation dosimetric ap- proach: outcome and validation in 85 patients. $\mathrm{J}$ Nucl Med 1992;33:1132-6.

7. de Klerk JM, de Keizer B, Zelissen PMJ, Lips CM, Koppeschaar HP. Fixed dosage of 131-I for remnant ablation in patients with differentiated thyroid carcinoma without pre-ablative diagnostic 131-I scintigraphy. Nucl Med Commun 2000;21:529 32.

8. Grigsby P. Cost minimization analysis and utility of pretreatment and posttreatment total body iodine131 scans in patients with thyroid carcinoma. Cancer 1998;82:931-5.

9. Albino CC, Takahashi MH, Senhorini Jr S, Graf H. Inquérito sobre o uso do iodo-131 no Brasil. Arq Bras Endocrinol Metab 2001;45:558-62.

10. Arslan N, Ilgan S, Serdengecti M, et al. Post-surgical ablation of thyroid remnants with high-dose (131)I in patients with differentiated thyroid carcinoma. Nucl Med Commun 2001;22:1021-7.

11. Morris LF, Waxman AD, Braunstein GD. Thyroid stunning. Thyroid 2003;13:333-40.

12. McDougall IR. $74 \mathrm{MBq}$ radioiodine I-131 does not prevent uptake of therapeutic doses of I-131 (i.e. it does not cause stunning) in differentiated thyroid cancer. Nucl Med Commun 1997;18:505-12.

13. Cholewinski SP, Yoo KS, Klieger PS, O'Mara RE. Absence of thyroid stunning after diagnostic wholebody scanning with $185 \mathrm{MBq}$ I-131. J Nucl Med 2000;41:1198-202.

14. Morris LF, Waxman AD, Braunstein GD. The nonimpact of thyroid stunning: remnant ablation rates in I-131-scanned and nonscanned individuals. J Clin Endocrinol Metab 2001;86:3507-11.

15. Van Wyngaarden M, McDougall IR. What is the role of $1100 \mathrm{MBq}(<30 \mathrm{mCi})$ radioiodine ${ }^{131} \mathrm{I}$ in the treatment of patients with differentiated thyroid cancer? Nucl Med Commun 1996;17:199-207.

16. Leung SF, Law MW, Ho SK. Efficacy of low-dose iodine-131 ablation of post-operative thyroid remnants: a study of 69 cases. Br J Radiol 1992;65: 905-9.

17. DeGroot LJ, Reilly M. Comparison of 30- and 50$\mathrm{mCi}$ doses of iodine-131 for thyroid ablation. Ann Intern Med 1982;96:51-3.

18. Doi SA, Woodhouse NJ. Ablation of the thyroid remnant and ${ }^{131} \mathrm{I}$ dose in differentiated thyroid cancer. Clin Endocrinol (Oxf) 2000;52:765-73.

19. Roos DE, Smith JG. Randomized trials on radioactive iodine ablation of thyroid remnants for thyroid carcinoma - a critique. Int J Radiat Oncol Biol Phys 1999;44:493-5.

20. Creutzig H. High or low dose radioiodine ablation of thyroid remnants? Eur J Nucl Med 1987;12: 500-2.

21. Johansen K, Woodhouse NJY, Odugbesan O. Comparison of $1073 \mathrm{MBq}$ and $3700 \mathrm{MBq}$ iodine-131 in postoperative ablation of residual thyroid tissue in patients with differentiated thyroid cancer. J Nucl Med 1991;32:252-4.

22. Lin JD, Kao PF, Chao TC. The effects of radioactive iodine in thyroid remnant ablation and treatment of well differentiated thyroid carcinoma. $\mathrm{Br}$ J Radiol 1998;71:307-13.

23. Bal CS, Kumar A, Pant GS. Radioiodine dose for remnant ablation in differentiated thyroid cancer: a randomized clinical trial in 509 patients. J Clin Endocrinol Metab 2004;89:1666-73. 